\title{
Study on New Doubly-periodic Solutions of two Coupled Nonlinear Wave Equations in Complex and Real Fields
}

\author{
Zhenya Yan \\ Key Laboratory of Mathematics Mechanization, Institute of systems Science, AMSS, \\ Chinese Academy of Sciences, Beijing 100080, P.R. China
}

Reprint requests to Dr.Z.Y.; E-mail: zyyan@mmrc.iss.ac.cn

Z. Naturforsch. 59a, 29 -34 (2004); received September 29, 2003

In this paper, new doubly-periodic solutions in terms of Weierstrass elliptic functions are investigated for the coupled nonlinear Schrödinger equation and systems of two coupled real scalar fields. Solitary wave solutions are also given as simple limits of doubly periodic solutions. - PACS: 03.40.Kf; 02.30Ik

Key words: Nonlinear Wave Equations; Weierstrass Elliptic Function; Doubly-periodic Solution; Solitary Wave Solution; Symbolic Computation.

\section{Introduction}

It is important for practical applications to investigate exact solutions in soliton theory. Presently much attention is paid to doubly periodic solutions of nonlinear wave equations[1-6,13]. In this paper we consider the following two coupled systems of nonlinear evolution equations:

A) The coupled nonlinear Schrodinger equations [7]

$$
\begin{array}{r}
i \frac{\partial}{\partial \zeta} \Psi_{1}+\frac{1}{2} \frac{\partial^{2}}{\partial \tau^{2}} \Psi_{1}+\frac{1}{2} \frac{\partial^{2}}{\partial \xi^{2}} \Psi_{1}+\left(\left|\Psi_{1}\right|^{2}+2\left|\Psi_{2}\right|^{2}\right) \Psi_{1}=0 \\
i \frac{\partial}{\partial \zeta} \Psi_{2}+\frac{1}{2} \frac{\partial^{2}}{\partial \xi^{2}} \Psi_{2}+\left(\left|\Psi_{2}\right|^{2}+2\left|\Psi_{1}\right|^{2}\right) \Psi_{2}=0, \quad(1)
\end{array}
$$

where $\zeta$ is the longitudinal coordinate, while $\tau$ and $\xi$ are the temporal and the transverse spatial coordinate. Pietrzyk [7] used the variational and numerical method to show that (1) admitts oscillating, self-trapped solutions.

(B) The system of two coupled real scalar fields [8-10]

$$
\begin{aligned}
& \phi_{t t}-\phi_{x x}+2 \lambda^{2} \phi^{3}-2 \lambda^{2} \sigma^{2} \phi+(\lambda+\mu) \phi \chi^{2}=0, \\
& \chi_{t t}-\chi_{x x}+\frac{1}{2} \mu^{2} \chi^{3}-\lambda \mu \sigma^{2} \chi+(\lambda+\mu) \phi^{2} \chi=0,
\end{aligned}
$$

which was derived from the potential form

$$
\begin{aligned}
U(\phi, \chi)= & \frac{1}{2} \lambda^{2}\left(\phi^{2}-\sigma^{2}\right)^{2}+\frac{1}{2} \lambda \mu\left(\phi^{2}-\sigma^{2}\right) \chi^{2} \\
& +\frac{1}{8} \mu^{2} \chi^{4}+\frac{1}{2} \mu^{2} \chi^{2} \phi^{2} .
\end{aligned}
$$

Knyzazev and Goncharenko [8] used two transformations to obtain one-kink and two-kink solutions.

Recently we developed a powerful Weierstrass elliptic function expansion method and its general form in terms of Weierstrass elliptic functions [11,12], which was applied to seek new doubly periodic solutions of some nonlinear wave equations [14]. This transformation is more general than the one due to Porubov [1], To the best of our knowledge, the doubly periodic solutions of the two systems (1) and (2) were not studied before. In this paper we extend the method to derive its doubly periodic solutions.

Firstly we simply introduce the algorithm as follows: For a given nonlinear evolution equation, $F\left(u, u_{t}, u_{x}, u_{x t}, \ldots\right)=0$, we seek its travelling wave solutions $u(x, t)=u(\xi), \xi=k(x-c t)$ in the form

$$
\begin{aligned}
u(\xi)=u\left(\wp\left(\xi ; g_{2}, g_{3}\right)\right) & \\
=a_{0}+\sum_{i=1}^{n}( & a_{i}\left[A \wp\left(\xi ; g_{2}, g_{3}\right)+B\right]^{\frac{i}{2}} \\
& \left.\quad+b_{i}\left[A \wp\left(\xi ; g_{2}, g_{3}\right)+B\right]^{-\frac{i}{2}}\right),
\end{aligned}
$$

where $n, A \neq 0, B, a_{0}, a_{i}, b_{i}$ are parameters to be determined later, and $\wp\left(\xi ; g_{2}, g_{3}\right)$ is the Weierstrass elliptic function satisfying the nonlinear ordinary differential equation

$$
[\wp(\xi)]^{2}=4 \wp^{3}(\xi)-g_{2} \wp(\xi)-g_{3},
$$


derivative of $\wp$ in the form

$$
[\wp(\xi)]^{\prime \prime}=6 \wp^{2}(\xi)-\frac{1}{2} g_{2},
$$

which is useful to solve the following nonlinear wave equations.

By the leading order analysis we determine the parameter $n$. And then we substitute (4) into the function $F$ along with (5) and (6) and get a complicated expression. Finally we take the numerator of the expression to get a polynomial about $\wp^{\prime i} \wp^{j}(\sqrt{A \wp+B})^{s}(i, s=$ $0,1 ; j=0,1,2, \ldots)$. Setting their coefficients to zero yields a system of algebraic equations about the unknowns. Solving the system, if available, can determine these unknowns. Thus we can obtain the doublyperiodic solutions of the given equation in terms of Weierstrass elliptic functions.

\section{The Coupled Nonlinear Schrödinger Equations}

To use the above method to seek doubly periodic solutions of (1), we reduce (1) to a system of nonlinear ordinary differential equations. We make the gauge transformations

$$
\begin{aligned}
& \Psi_{1}(\xi, \zeta, \tau)=\psi_{1}(X) \exp \left(i Y_{1}\right), \\
& \Psi_{2}(\xi, \zeta, \tau)=\psi_{2}(X) \exp \left(i Y_{2}\right), \quad i=\sqrt{-1}, \\
& X=k(\xi+l \zeta+\lambda \tau), Y_{j}=\alpha_{j} \xi+\beta_{j} \zeta+\gamma_{j} \tau, \quad j=1,2, \\
& \text { where } k \neq 0, l, \lambda, \alpha_{i}, \beta_{i} \text {, and } \gamma_{i} \text { are real parameters to } \\
& \text { be determined later. Substituting (7) into (1) yields } \\
& i\left(k l+\lambda k \gamma_{1}+k \alpha_{1}\right) \psi_{1}^{\prime}+\frac{1}{2} k^{2}\left(1+\lambda^{2}\right) \psi_{1}^{\prime \prime} \\
& \quad-\left(\beta_{1}+\frac{1}{2} \gamma_{1}^{2}+\frac{1}{2} \alpha_{1}^{2}\right) \psi_{1}+\psi_{1}^{3}+2 \psi_{2}^{2} \psi_{1}=0, \quad \text { (8a) } \\
& \quad+\psi_{2}^{3}+2 \psi_{1}^{2} \psi_{2}=0 .
\end{aligned}
$$

Setting the real and imaginary parts of (8) to zero yields the relationships

$$
\begin{gathered}
\alpha_{1}=-\left(l+\lambda \gamma_{1}\right), \quad \alpha_{2}=-l, \\
\frac{1}{2} k^{2}\left(1+\lambda^{2}\right) \psi_{1}^{\prime \prime}-\left(\beta_{1}+\frac{1}{2} \gamma_{1}^{2}+\frac{1}{2} \alpha_{1}^{2}\right) \psi_{1} \\
+\psi_{1}^{3}+2 \psi_{2}^{2} \psi_{1}=0,
\end{gathered}
$$

$$
\frac{1}{2} k^{2} \psi_{2}^{\prime \prime}-\left(\beta_{2}+\frac{1}{2} \alpha_{2}^{2}\right) \psi_{2}+\psi_{2}^{3}+2 \psi_{1}^{2} \psi_{2}=0
$$

To conveniently consider the system (10) we make the ansatz $\psi_{2}=C \psi_{1}(C$ constant $)$. Then the system (10) holds provided that

$$
\frac{\lambda^{2}+1}{C}=\frac{\beta_{1}+\frac{1}{2} \gamma_{1}^{2}+\frac{1}{2} \alpha_{1}^{2}}{C\left(\beta_{2}+\frac{1}{2} \alpha_{2}^{2}\right)}=\frac{1+2 C^{2}}{C^{3}+2 C},
$$

which leads to these relationships

$$
\begin{aligned}
& C^{2}=\frac{2 \lambda^{2}+1}{1-\lambda^{2}}, \\
& \beta_{1}=\left(\lambda^{2}+1\right)\left(\beta_{2}+\frac{1}{2} \alpha_{2}^{2}\right)-\frac{1}{2} \gamma_{1}^{2}-\frac{1}{2} \alpha_{1}^{2}, \\
& \lambda^{2} \neq 1 .
\end{aligned}
$$

Therefore under the ansatz $\psi_{2}=C \psi_{1}$ and the conditions (12), (10) reduces to one equation:

$$
\frac{1}{2} k^{2} \psi_{1}^{\prime \prime}-\left(\beta_{2}+\frac{1}{2} l^{2}\right) \psi_{1}+\frac{3}{1-\lambda^{2}} \psi_{1}^{3}=0 .
$$

Substituting (4) into (13) along with (5) and (6), and using the leading order analysis we know that $n=1$. Thus we assume that (13) has the solution

$$
\begin{aligned}
\psi_{1}(X)= & \psi_{1}\left(\wp\left(X ; g_{2}, g_{3}\right)\right) \\
= & a_{0}+a_{1} \sqrt{A \wp\left(X ; g_{2}, g_{3}\right)+B} \\
& +\frac{b_{1}}{\sqrt{A \wp\left(X ; g_{2}, g_{3}\right)+B}},
\end{aligned}
$$

where $\wp\left(X ; g_{2}, g_{3}\right)$ satisfies

$$
[\wp(X)]^{\prime 2}=4 \wp^{3}(\xi)-g_{2} \wp(X)-g_{3},
$$

or

$$
[\wp(X)]^{\prime \prime}=6 \wp^{3}(X)-\frac{1}{2} g_{2} .
$$

With the aid of Maple, substituting (14) into (13) along with (15) and (16), and equating the coefficients of the terms $\wp^{j}(\sqrt{A \wp+B})^{i}(i=0,1 ; j=0,1,2,3,4,5)$, we get the system of algebraic equations

(9) $\frac{3}{1-\lambda^{2}}\left(12 a_{1}^{2} a_{0} B^{4}+24 a_{1} b_{1} a_{0} B^{3}+4 a_{0}^{3} B^{3}+12 b_{1}^{2} a_{0} B^{2}\right)$

$$
\begin{aligned}
& -4 a_{0} B^{3}\left(\beta_{2}+\frac{1}{2} l^{2}\right)=0, \\
& 12 a_{1}^{2} a_{0} A^{4} \frac{3}{1-\lambda^{2}}=0,
\end{aligned}
$$


Z. Yan · Solutions of two Coupled Nonlinear Wave Equations in Complex and Real Fields

$$
\begin{aligned}
& \frac{3}{1-\lambda^{2}}\left(12 a_{0}^{3} B^{2} A+24 b_{1}^{2} a_{0} A B+72 a_{1} b_{1} a_{0} B^{2} A+48 a_{1}^{2} a_{0} B^{3} A\right)-12 a_{0} A B^{2}\left(\beta_{2}+\frac{1}{2} l^{2}\right)=0, \\
& \frac{3}{1-\lambda^{2}}\left(24 a_{1} b_{1} a_{0} A^{3}+4 a_{0}^{3} A^{3}+48 a_{1}^{2} a_{0} A^{3} B\right)-4 a_{0} A^{3}\left(\beta_{2}+\frac{1}{2} l^{2}\right)=0, \\
& \frac{3}{1-\lambda^{2}}\left(72 a_{1} b_{1} a_{0} B A^{2}+12 a_{0}^{3} B A^{2}+72 a_{1}^{2} a_{0} B^{2} A^{2}+12 b_{1}^{2} a_{0} A^{2}\right)-12 a_{0} A^{2} B\left(\beta_{2}+\frac{1}{2} l^{2}\right)=0, \\
& \frac{3}{1-\lambda^{2}}\left(12 b_{1} a_{0}^{2} B^{3}+12 b_{1} a_{1}^{2} B^{4}+12 a_{1} a_{0}^{2} B^{4}+12 a_{1} b_{1}^{2} B^{3}+4 a_{1}^{3} B^{5}+4 b_{1}^{3} B^{2}\right) \\
& +\frac{1}{2} k^{2}\left(b_{1} A g_{2} B^{2}-3 b_{1} A^{2} g_{3} B+a_{1} A^{2} g_{3} B^{2}-a_{1} A g_{2} B^{3}\right)-\left(4 b_{1} B^{3}+4 a_{1} B^{4}\right)\left(\beta_{2}+\frac{1}{2} l^{2}\right)=0, \\
& \frac{3}{1-\lambda^{2}}\left(20 a_{1}^{3} A^{4} B+12 a_{1} a_{0}^{2} A^{4}+12 b_{1} a_{1}^{2} A^{4}\right)-4 a_{1} A^{4}\left(\beta_{2}+\frac{1}{2} l^{2}\right)+14 k^{2} a_{1} A^{3} B=0, \\
& 4 a_{1}^{3} A^{5} \frac{3}{1-\lambda^{2}}+4 k^{2} a_{1} A^{4}=0, \\
& \frac{3}{1-\lambda^{2}}\left(8 b_{1}^{3} B A+20 a_{1}^{3} A B^{4}+48 b_{1} a_{1}^{2} A B^{3}+36 b_{1} a_{0}^{2} B^{2} A+48 a_{1} a_{0}^{2} A B^{3}+36 a_{1} b_{1}^{2} A B^{2}\right) \\
& -\frac{1}{2} k^{2}\left(b_{1} A^{2} g_{2} B+2 a_{1} A^{2} g_{2} B^{2}+3 b_{1} A^{3} g_{3}-2 a_{1} A^{3} g_{3} B\right)=0, \\
& \frac{3}{1-\lambda^{2}}\left(12 a_{1} b_{1}^{2} A^{3}+48 a_{1} a_{0}^{2} A^{3} B+40 a_{1}^{3} A^{3} B^{2}+48 b_{1} a_{1}^{2} A^{3} B+12 b_{1} a_{0}^{2} A^{3}\right) \\
& -\left(\beta_{2}+\frac{1}{2} l^{2}\right)\left(4 b_{1} A^{3}+16 a_{1} A^{3} B\right)-k^{2}\left(6 b_{1} A^{2} B-16 a_{1} A^{2} B^{2}\right)=0, \\
& \frac{3}{1-\lambda^{2}}\left(72 a_{1} a_{0}^{2} A^{2} B^{2}+72 b_{1} a_{1}^{2} A^{2} B^{2}+36 a_{1} b_{1}^{2} A^{2} B+40 a_{1}^{3} A^{2} B^{3}+4 b_{1}^{3} A^{2}+36 b_{1} a_{0}^{2} B A^{2}\right) \\
& -\left(\beta_{2}+\frac{1}{2} l^{2}\right)\left(24 a_{1} A^{2} B^{2}+12 b_{1} A^{2} B\right)+\frac{1}{2} k^{2}\left(a_{1} A^{4} g_{3}+12 a_{1} A B^{3}-a_{1} A^{3} g_{2} B-2 b_{1} A^{3} g_{2}-12 b_{1} A B^{2}\right)=0 .
\end{aligned}
$$

It is complicated to solve the system by hand, but with the aid of Maple we easily get three nontrivial solutions.

Case 1:

$$
\begin{aligned}
& a_{0}=b_{1}=0, \quad a_{1} \neq 0, \\
& A=\frac{k^{2}\left(\lambda^{2}-1\right)}{3 a_{1}^{2}}, \quad B=\frac{\left(2 \beta_{2}+l^{2}\right)\left(1-\lambda^{2}\right)}{9 a_{1}^{2}}, \\
& g_{3}=\frac{1}{27 k^{6}}\left[4\left(2 \beta_{2}+l^{2}\right)^{3}-9 k^{4} g_{2}\left(2 \beta_{2}+l^{2}\right)\right],
\end{aligned}
$$

Case 2:

$$
\begin{aligned}
& a_{0}=a_{1}=0, \quad b_{1} \neq 0, \quad A=-\frac{3 k^{2} B}{2 \beta_{2}+l^{2}}, \\
& g_{2}=\frac{1}{3 k^{4} B}\left[-\frac{12 b_{1}^{2}\left(2 \beta_{2}+l^{2}\right)}{1-\lambda^{2}}+4 B\left(2 \beta_{2}+l^{2}\right)^{2}\right],
\end{aligned}
$$

$g_{3}=\frac{1}{27 k^{6} B}\left[\frac{36 b_{1}^{2}\left(2 \beta_{2}+l^{2}\right)^{2}}{1-\lambda^{2}}-8 B\left(2 \beta_{2}+l^{2}\right)^{3}\right]$.

Case 3:

$$
\begin{aligned}
a_{0} & =0, \quad a_{1} \neq 0, \quad b_{1} \neq 0, \\
A & =-\frac{1}{3} k^{2}\left(1-\lambda^{2}\right), \\
B & =-\frac{-\left(2 \beta_{2}+l^{2}\right)\left(1-\lambda^{2}\right)+18 a_{1} b_{1}}{9 a_{1}^{2}}, \\
g_{2}=\frac{1}{3 k^{4}}\left[\frac{1188 a_{1}^{2} b_{1}^{2}}{\left(1-\lambda^{2}\right)^{2}}-\frac{144 a_{1} b_{1}\left(2 \beta_{2}+l^{2}\right)}{1-\lambda^{2}}\right. & \left.+4\left(2 \beta_{2}+l^{2}\right)^{2}\right],
\end{aligned}
$$




$$
g_{3}=\frac{1}{27 k^{6}}\left[\frac{40824 a_{1}^{3} b_{1}^{3}}{\left(1-\lambda^{2}\right)^{3}}-\frac{7452 a_{1}^{2} b_{1}^{2}\left(2 \beta_{2}+l^{2}\right)}{\left(1-\lambda^{2}\right)^{2}}+\frac{432 a_{1} b_{1}\left(2 \beta_{2}+l^{2}\right)^{2}}{1-\lambda^{2}}+8\left(2 \beta_{2}+l^{2}\right)^{3}\right] .
$$

Therefore we get three types of envelopment doubly periodic solutions of (1) in terms of Weierstrass elliptic function:

Family 1:

$$
\begin{aligned}
& \Psi_{1}= \pm \sqrt{\frac{k^{2}\left(\lambda^{2}-1\right)}{3} \wp\left(X ; g_{2}, g_{3}\right)+\frac{\left(2 \beta_{2}+l^{2}\right)\left(1-\lambda^{2}\right)}{9}} \exp \left(i Y_{1}\right), \\
& \Psi_{2}= \pm \sqrt{-\frac{k^{2}\left(2 \lambda^{2}+1\right)}{3} \wp\left(X ; g_{2}, g_{3}\right)+\frac{\left(2 \lambda^{2}+1\right)\left(2 \beta_{2}+l^{2}\right)}{9}} \exp \left(i Y_{2}\right),
\end{aligned}
$$

where $\alpha_{1}, \beta_{1}$ satisfy (9) and (12), $g_{3}$ is determined by (18), and $X=k(\xi+l \zeta+\lambda \tau), Y_{j}=\alpha_{j} \xi+\beta_{j} \zeta+\gamma_{j} \tau$ $(j=1,2)$.

Family 2:

$$
\Psi_{1}=\frac{b_{1} \exp \left(i\left(\alpha_{1} \xi+\beta_{1} \zeta+\gamma_{1} \tau\right)\right)}{\sqrt{-\frac{3 k^{2} B}{2 \beta_{2}+l^{2}} \wp\left(k(\xi+l \zeta+\lambda \tau) ; g_{2}, g_{3}\right)+B}}, \quad \Psi_{2}=\frac{b_{1} \sqrt{\frac{2 \lambda^{2}+1}{1-\lambda^{2}}} \exp \left(i\left(\alpha_{2} \xi+\beta_{2} \zeta+\gamma_{2} \tau\right)\right)}{\sqrt{-\frac{3 k^{2} B}{2 \beta_{2}+l^{2}} \wp\left(k(\xi+l \zeta+\lambda \tau) ; g_{2}, g_{3}\right)+B}},
$$

where $b_{1} \neq 0, B, l, k, \alpha_{2}, \beta_{2}, \gamma_{1}, \gamma_{2}$ are constants, $\alpha_{1}, \beta_{1}$ satisfy (9) and (12), $g_{2}, g_{3}$ is determined by (19). Family 3:

$$
\begin{aligned}
& \Psi_{1}=-\frac{\left[3 k^{2}\left(1-\lambda^{2}\right) \wp\left(X ; g_{2}, g_{3}\right)+\left(2 \beta_{2}+l^{2}\right)\left(1-\lambda^{2}\right)-9 a_{1} b_{1}\right] \exp \left(i Y_{1}\right)}{3 \sqrt{-3 k^{2}\left(1-\lambda^{2}\right) \wp\left(X ; g_{2}, g_{3}\right)-\left(2 \beta_{2}+l^{2}\right)\left(1-\lambda^{2}\right)-18 a_{1} b_{1}}} \\
& \Psi_{2}= \pm \sqrt{\frac{2 \lambda^{2}+1}{1-\lambda^{2}}} \frac{\left[3 k^{2}\left(1-\lambda^{2}\right) \wp\left(X ; g_{2}, g_{3}\right)+\left(2 \beta_{2}+l^{2}\right)\left(1-\lambda^{2}\right)-9 a_{1} b_{1}\right] \exp \left(i Y_{2}\right)}{3 \sqrt{-3 k^{2}\left(1-\lambda^{2}\right) \wp\left(X ; g_{2}, g_{3}\right)-\left(2 \beta_{2}+l^{2}\right)\left(1-\lambda^{2}\right)-18 a_{1} b_{1}}}
\end{aligned}
$$

where $a_{1} \neq 0, b_{1} \neq 0, B, l, k, \alpha_{2}, \beta_{2}, \gamma_{1}, \gamma_{2}$ are constants, $X=k(\xi+l \zeta+\lambda \tau), Y_{j}=\alpha_{j} \xi+\beta_{j} \zeta+\gamma_{j} \tau(j=1,2)$, $\alpha_{1}, \beta_{1}$ satisfy (9) and (12), $g_{2}, g_{3}$ is determined by (20).

In order to better understand the solution (21), we rewrite (21) in the form of Jacobi elliptic functions:

$$
\begin{aligned}
& \Psi_{11}= \pm \sqrt{\frac{k^{2}\left(\lambda^{2}-1\right)}{3}\left[e_{2}-\left(e_{2}-e_{3}\right) \operatorname{cn}^{2}(\mu X ; m)\right]+\frac{\left(2 \beta_{2}+l^{2}\right)\left(1-\lambda^{2}\right)}{9}} \exp \left(i Y_{1}\right), \\
& \Psi_{21}= \pm \sqrt{-\frac{k^{2}\left(2 \lambda^{2}+1\right)}{3}\left[e_{2}-\left(e_{2}-e_{3}\right) \mathrm{cn}^{2}(\mu X ; m)\right]+\frac{\left(2 \lambda^{2}+1\right)\left(2 \beta_{2}+l^{2}\right)}{9}} \exp \left(i Y_{2}\right),
\end{aligned}
$$

where $\mu=\sqrt{e_{1}-e_{3}}, m^{2}=\left(e_{2}-e_{3}\right) /\left(e_{1}-e_{3}\right)$ is the modulus of the Jacobi elliptic function, $e_{i}\left(i=1,2,3 ; e_{1}>\right.$ $\left.e_{2}>e_{3}\right)$ are three roots of the cubic equation $4 z^{3}-g_{2} z-g_{3}=0$.

Because when $m \rightarrow 1$, i.e., $e_{2} \rightarrow e_{1}, \operatorname{cn}(\mu X ; m) \rightarrow \operatorname{sech}(\mu X)$, thus the envelopment solitary wave profile can be written in the form

$$
\Psi_{12}= \pm \sqrt{\frac{k^{2}\left(\lambda^{2}-1\right)}{3}\left[e_{2}-\left(e_{2}-e_{3}\right) \operatorname{sech}^{2}(\mu X ; m)\right]+\frac{\left(2 \beta_{2}+l^{2}\right)\left(1-\lambda^{2}\right)}{9}} \exp \left(i Y_{1}\right),
$$




$$
\Psi_{22}= \pm \sqrt{-\frac{k^{2}\left(2 \lambda^{2}+1\right)}{3}\left[e_{2}-\left(e_{2}-e_{3}\right) \operatorname{sech}^{2}(\mu X ; m)\right]+\frac{\left(2 \lambda^{2}+1\right)\left(2 \beta_{2}+l^{2}\right)}{9}} \exp \left(i Y_{2}\right) .
$$

When $X \rightarrow \infty$, we know that $\left|\Psi_{12}\right|$ and $\left|\Psi_{22}\right|$ tend to the two constants

$$
\pm \frac{1}{3} \sqrt{3 e_{2} k^{2}\left(\lambda^{2}-1\right)+\left(2 \beta_{2}+l^{2}\right)\left(1-\lambda^{2}\right)}, \pm \frac{1}{3} \sqrt{\left(2 \lambda^{2}+1\right)\left(-3 e_{2} k^{2}+2 \lambda^{2}+1\right)},
$$

respectively.

For the solutions (19) and (20), we can also rewrite them by using Jacobi elliptic functions so that the corresponding envelopment solitary wave solutions in limiting cases are derived. Here we omit them. In addition, the Weierstrass elliptic function $\wp\left(X ; g_{2}, g_{3}\right)$ has other types of Jacobi elliptic functions, which lead to other types of envelop solitary wave solutions in limiting cases.

\section{The System of two Coupled Real Scalar \\ Fields (2)}

Similar to (1), according to the above method, when $\mu=2 \lambda$, we can also arrive at the doubly periodic solutions of (2):

Family 1:

$\phi_{1}= \pm \sqrt{\frac{k^{2}\left(1-c^{2}\right)}{4 \lambda^{2}} \wp\left(k(x-c t) ; g_{2}, g_{3}\right)+\frac{a^{2}}{6}}, \varepsilon= \pm 1$,

$\chi_{1}= \pm \varepsilon \sqrt{\frac{k^{2}\left(1-c^{2}\right)}{4 \lambda^{2}} \wp\left(k(x-c t) ; g_{2}, g_{3}\right)+\frac{a^{2}}{6}}$ where $k, c$ are constants, and

$g_{3}=\frac{1}{27 k^{6}\left(c^{2}-1\right)^{3}}\left[32 \lambda^{6} a^{6}-18 g_{2} \lambda^{2} a^{2} k^{4}\left(c^{2}-1\right)^{2}\right]$.

Family 2:

$$
\begin{aligned}
& \phi_{2}=\frac{b_{1}}{\sqrt{\frac{3 k^{2}\left(1-c^{2}\right) B}{2 \lambda^{2} a^{2}} \wp\left(k(x-c t) ; g_{2}, g_{3}\right)+B}}, \\
& \chi_{2}=\frac{b_{1} \varepsilon}{\sqrt{\frac{3 k^{2}\left(1-c^{2}\right) B}{2 \lambda^{2} a^{2}} \wp\left(k(x-c t) ; g_{2}, g_{3}\right)+B}},
\end{aligned}
$$

where $k, c, B, b_{1} \neq 0$ are constants, and

$$
\begin{aligned}
& g_{2}=\frac{-16 \lambda^{4} a^{4} b_{1}^{2}+8 \lambda^{4} a^{4} B}{B k^{4}\left(1-c^{2}\right)^{2}}, \\
& g_{3}=\frac{192 \lambda^{6} a^{4} b_{1}^{2}-64 \lambda^{6} a^{6} B}{27 B k^{6}\left(1-c^{2}\right)^{3}} .
\end{aligned}
$$

Family 3:

$$
\begin{aligned}
& \phi_{3}=\frac{-3 k^{2}\left(c^{2}-1\right) \wp\left(k(x-c t) ; g_{2}, g_{3}\right)+2 \lambda^{2}\left(a^{2}-6 a_{1} b_{1}\right)}{2 \sqrt{-3 k^{2}\left(c^{2}-1\right) \wp\left(k(x-c t) ; g_{2}, g_{3}\right)+2 \lambda^{2}\left(a^{2}-12 a_{1} b_{1}\right)}}, \\
& \chi_{3}=\frac{-3 k^{2}\left(c^{2}-1\right) \wp\left(k(x-c t) ; g_{2}, g_{3}\right)+2 \lambda^{2}\left(a^{2}-6 a_{1} b_{1}\right)}{2 \varepsilon \sqrt{-3 k^{2}\left(c^{2}-1\right) \wp\left(k(x-c t) ; g_{2}, g_{3}\right)+2 \lambda^{2}\left(a^{2}-12 a_{1} b_{1}\right)}},
\end{aligned}
$$

where $k, c, B, a_{1} \neq 0, b_{1} \neq 0$ are constants, and

$$
\begin{aligned}
& g_{2}=\frac{1}{3 k^{4}\left(c^{2}-1\right)^{2}}\left(16 \lambda^{4} a^{4}-384 a_{1} b_{1} \lambda^{4} a^{2}+2112 a_{1}^{2} b_{1}^{2} \lambda^{4}\right), \\
& g_{3}=\frac{1}{27 k^{6}\left(c^{2}-1\right)^{3}}\left(-64 \lambda^{6} a^{6}+2304 a_{1} b_{1} \lambda^{6} a^{4}-26496 a_{1}^{2} b_{1}^{2} \lambda^{6} a^{2}+96768 a_{1}^{3} b_{1}^{3} \lambda^{6}\right) .
\end{aligned}
$$




\section{Conclusions}

In summary, we have extended the Weierstrass elliptic function expansion method [14] to two systems (1) and (2). With the aid of symbolic computation we obtained three types of doubly peri-

[1] A. V. Porubov, Phys. Lett. A 221, 391 (1996).

[2] A. V. Porubov and M. G. Velarde, J. Math. Phys. 40, 884 (1999).

[3] Z. Y. Yan, Commun. Theor. Phys. 38, 143 (2002); 38, 400 (2002); 39, 144 (2003).

[4] Z. Y. Yan, Chaos, Solitons, and Fractals 15, 575 (2003).

[5] Z. Y. Yan, Comput. Phys. Commun. 148, 30 (2002).

[6] Z. Y. Yan, Chaos, Solitons and Fractals, 16, 291 (2003).

[7] M. E. Pietrzyk, Rep. Math. Phys. 46, 225 (2000). odic solutions. In limiting cases solitary wave solutions are obtained. These solutions may be useful to explain some physical phenomena. These need to be studied. Moreover the method can also be extended to some other nonlinear mathematical physics equations.

[8] M. A. Knyazev and E. A. Goncharenko, Rep. Math. Phys. 46, 119 (2000).

[9] D. Bazeia, et al., Phys. Rev. A 54, 2943 (1996).

[10] K. Narita, J. Phys. Soc. Japan 54, 1249 (1985).

[11] D.F. Lawden, Elliptic Functions and Applications, Springer-Verlag, New York 1989.

[12] E. T. Whittaker and G. N. Watson, A Course of Modern Analysis. Cambridge University Press, London 1950.

[13] Z. Y. Yan, J. Phys. A 36, 1961 (2003).

[14] Z. Y. Yan, (to be sumbitted). 